\title{
Corrigendum: Monogamy and haplodiploidy act in synergy to promote the evolution of eusociality
}

Lutz Fromhage \& Hanna Kokko

Nature Communications 2:397 doi: 10.1038/ncomms1410 (2011); Published 19 Jul 2011; Updated 5 Feb 2013

This Article contains typographical errors in Equations (6) and (7), where terms were inadvertently left out. These errors were not present in the analysed model itself, so did not affect the results.

Equation (6) is missing the term $(n-1) d_{\mathrm{w}}$, representing worker mortality in colonies of size $n$, and should read:

$$
\mathrm{d} X_{k, n} / \mathrm{d} t=X_{k, n-1} \phi b(n-1) w_{k}+\mathrm{d}_{w} n X_{k, n+1}-X_{k, n}\left(d(n)+(n-1) d_{w}+\phi b(n) w_{k}\right) .
$$

Equation (7) should read:

$$
\mathrm{d} X_{k, N} / \mathrm{d} t=X_{k, N-1} \phi b(N-1) w_{k}-X_{k, N}\left(d(N)+(N-1) d_{w}\right) .
$$

In addition, some of the entries in Supplementary Table S1, lines 4 and 5, were inadvertently exchanged. The correct version of the Table appears below.

\begin{tabular}{clllccccc}
\hline$k$ & female & male & $\chi_{\mathrm{k}}$ & $v_{\mathrm{AA}, \mathrm{k}}$ & $v_{\mathrm{Aa}, \mathrm{k}}$ & $v_{\mathrm{aa}, \mathrm{k}}$ & $v_{\mathrm{A}, \mathrm{k}}$ & $v_{\mathrm{a}, \mathrm{k}}$ \\
\hline 1 & $\mathrm{AA}$ & $\mathrm{A}$ & $x_{\mathrm{AA}} \beta y_{\mathrm{A}}$ & 1 & 0 & 0 & 1 & 0 \\
2 & $A A$ & $a$ & $x_{\mathrm{AA}} \beta y_{\mathrm{a}}$ & 0 & 1 & 0 & 1 & 0 \\
3 & $A a$ & $\mathrm{~A}$ & $x_{\mathrm{Aa}} \beta y_{\mathrm{A}}$ & $1 / 2$ & $1 / 2$ & 0 & $1 / 2$ & $1 / 2$ \\
4 & $A a$ & $a$ & $x_{\mathrm{Aa}} \beta y_{\mathrm{a}}$ & 0 & $1 / 2$ & $1 / 2$ & $1 / 2$ & $1 / 2$ \\
5 & $a a$ & $\mathrm{~A}$ & $x_{\mathrm{a} a} \beta y_{\mathrm{A}}$ & 0 & 1 & 0 & 0 & 1 \\
6 & $a a$ & $a$ & $x_{\mathrm{aa}} \beta y_{\mathrm{a}}$ & 0 & 0 & 1 & 0 & 1 \\
\hline
\end{tabular}

